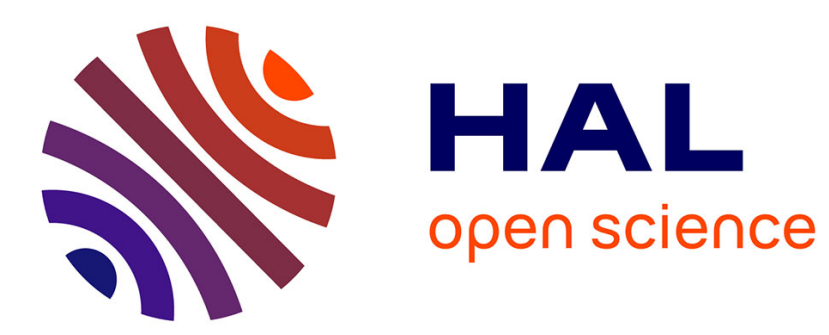

\title{
Fluage de la phase $\beta$ de l'Ag-Zn équiatomique
}

C. Lexcellent, C. Oytana

\section{- To cite this version:}

C. Lexcellent, C. Oytana. Fluage de la phase $\beta$ de l'Ag-Zn équiatomique. Revue de Physique Appliquée, 1978, 13 (4), pp.171-175. 10.1051/rphysap:01978001304017100 . jpa-00244436

\section{HAL Id: jpa-00244436 https://hal.science/jpa-00244436}

Submitted on 1 Jan 1978

HAL is a multi-disciplinary open access archive for the deposit and dissemination of scientific research documents, whether they are published or not. The documents may come from teaching and research institutions in France or abroad, or from public or private research centers.
L'archive ouverte pluridisciplinaire $\mathbf{H A L}$, est destinée au dépôt et à la diffusion de documents scientifiques de niveau recherche, publiés ou non, émanant des établissements d'enseignement et de recherche français ou étrangers, des laboratoires publics ou privés. 
Classification

Physics Abstracts

$80.00-81.40 \mathrm{~L}$

\title{
FLUAGE DE LA PHASE $\beta$ DE L'Ag-Zn ÉQUIATOMIQUE
}

\author{
C. LEXCELLENT et C. OYTANA \\ Laboratoire de Mécanique Appliquée (*), \\ Faculté des Sciences, 25030 Besançon Cedex, France
}

(Reçu le 18 juillet 1977, révisé le 21 novembre 1977, accepté le 5 janvier 1978)

\begin{abstract}
Résumé. - Etude du fluage de la phase $\beta$ équiatomique Ag-Zn. L'utilisation de l'équation de Dorn nécessite la mesure des vitesses de fluage stationnaire en fonction de la température $T$ et de la contrainte $\sigma$ et celle du module de Coulomb $G$. On compare l'énergie d'activation mesurée intervenant dans cette équation avec celle du coefficient de diffusion prévu par les cas extrêmes : régime stationnaire et régime homogène.
\end{abstract}

\begin{abstract}
Study of the $\beta$ phase of equiatomic Ag-Zn. The utilisation of Dorn equation demands the measurement of stationary creep rates versus temperature and stress $\sigma$ and the one of Coulomb modulus $G$. The measured activation energy which takes place in this equation is compared with that of the diffusion coefficient as calculated in the two extreme cases : the stationary and the homogeneous ones.
\end{abstract}

1. Introduction. - Le fluage des solutions solides concentrées n'a donné lieu qu'à un nombre réduit d'études expérimentales et pose un certain nombre de problèmes quant à son analyse [1].

Sherby et Burke [2] ont proposé une classification, assez contestable, des solutions solides reposant sur le coefficient de sensibilité de la vitesse de fluage $\stackrel{\circ}{\varepsilon}_{\mathbf{s}}$ à la contrainte appliquée $\sigma$ :

$$
n=\left(\frac{\partial \operatorname{Ln} \stackrel{\circ}{\varepsilon_{s}}}{\partial \operatorname{Ln} \sigma}\right)_{T}
$$

qui peut être de l'ordre de 3 à 3,5 (classe I) ou supérieure à cette valeur (classe II). Un certain nombre de méthodes ont été proposées pour déterminer la valeur d'une possible contrainte interne $\sigma_{i}$ qui conduirait à

$$
n^{\prime}=\left(\frac{\partial \operatorname{Ln} \stackrel{\circ}{\varepsilon}_{\mathrm{s}}}{\partial \operatorname{Ln}\left(\sigma-\sigma_{\mathrm{i}}\right)}\right)_{T} .
$$

Ces méthodes, souvent discutées, ont conduit à des valeurs de $\sigma_{\mathrm{i}}$ très faibles dans le cas du laiton $\beta$ [3]. La présence d'une éventuelle contrainte interne conduit à différentes définitions de l'enthalpie d'activation pour le fluage [4] et le problème peut se poser de savoir laquelle il convient de comparer à l'énergie d'activation des coefficients de diffusion de la solution solide.

Les résultats obtenus sur le laiton $\beta$ et d'autres solutions solides équiatomiques cubiques centrées condui-

(*) Laboratoire associé au C. N. R.S. sent systématiquement à un paramètre $n \simeq 3,5$. De même, les mesures de $\sigma_{i}$, par relaxation ou par décréments de contraintes en cours de fluage donnent dans ces cas des valeurs très faibles de $\sigma_{i}$. L'Ag-Zn qui se situe dans cette famille d'alliage ne devrait pas échapper à cette règle. Comme ces méthodes ne sont pas sûres, cela laisse espérer que le paramètre

$$
\Delta H=-\left(\frac{\partial \operatorname{Ln} \stackrel{\circ}{\varepsilon}_{\mathrm{S}}}{\partial \frac{1}{k T}}\right)_{\sigma}
$$

est celui qu'il faut comparer aux énergies de diffusion. (Les différentes définitions de Saxl et Kroupa [4] conduisent d'ailleurs au même résultat quand $\sigma_{\mathrm{i}}$ est faible.) Cet alliage présente également l'avantage d'avoir des coefficients de diffusion $D_{\mathrm{Ag}}$ et $D_{\mathrm{Zn}}$ mesurés aux radiotraceurs et d'énergie d'activation très différents rendant les comparaisons avec $\Delta H$ faciles.

Nous nous proposons de présenter les résultats d'expériences de fluage conduites en ce sens sur la phase $\beta$ de cet alliage.

2. Etude expérimentale. - 2.1 MÉTHode. - Les échantillons brut de filage ont été maintenus 2 heures à $475-485^{\circ} \mathrm{C}$ (en phase $\beta$ ) sous argon gazeux puis trempés dans l'eau glacée. La texture obtenue est constituée de gros grains $(\delta=500$ à $1000 \mu)$ de phase $\beta^{\prime}$ ordonnée.

Afin de passer de $\beta^{\prime}$ à une structure $\beta$ désordonnée et homogène, les éprouvettes trempées sont ensuite maintenues en température 1 heure à $495^{\circ} \mathrm{C}$ et 1 heure 
à la température d'essais. Les essais sont réalisés entre $280^{\circ} \mathrm{C}$ et $495^{\circ} \mathrm{C}$ c'est-à-dire jusqu'à $220^{\circ} \mathrm{C}$ au-dessus du point de transition de $\xi_{0} \rightarrow \beta$ (qui se situe à $274^{\circ} \mathrm{C}$ ).

La contrainte varie de $0,2 \times 10^{7} \mathrm{~N} / \mathrm{m}^{2}$ à $1,8 \times 10^{7} \mathrm{~N} / \mathrm{m}^{2}$. La machine de fluage utilisée comporte un chauffage par lit fluidisé. Cette technique permet d'obtenir une grande stabilité en température et un gradient thermique presque nul même pour les grandes déformations. La réalisation d'une came reposant sur le principe d'Andrade a permis de soumettre l'éprouvette à une contrainte constante dans le temps.

Le module de Coulomb $G$ est un paramètre important dans l'analyse du fluage et son évolution en fonction de la température peut conduire à des écarts importants dans la détermination de $\Delta H$. Il a été mesuré en dynamique, à quelques centaines de $\mathrm{Hz}$ par l'intermédiaire d'une éprouvette en vibrations forcées.

2.3 RÉSUltats EXPÉRIMENTAUX. - Les mesures du module de Coulomb ont donné une variation sensiblement linéaire de $\mathbf{G}$ avec :

$$
\begin{array}{lll}
G=1,086 \times 10^{10} \mathrm{~N} / \mathrm{m}^{2} & \text { à } & T=300^{\circ} \mathrm{C} \\
G=0,889 \times 10^{10} \mathrm{~N} / \mathrm{m}^{2} & \text { à } & T=500^{\circ} \mathrm{C} .
\end{array}
$$

- Les courbes présentent un fluage primaire de faible amplitude dont la durée décroît quand la température et la contrainte augmentent.
Le fluage stationnaire est très important, ce qui permet une mesure facile de $\stackrel{\circ}{\varepsilon}_{\text {s }}$.

La figure 1 montre l'évolution de la vitesse de fluage stationnaire en diagramme d'Arrhenius, à contrainte appliquée constante. Pour $\sigma<1,8 \times 10^{7} \mathrm{~N} / \mathrm{m}^{2}$, on trouve une énergie d'activation apparente de l'ordre de $21,5 \mathrm{kcal} / \mathrm{mole}$.

La figure 2 sur laquelle sont portées les isothermes de $\operatorname{Ln} \stackrel{\circ}{\varepsilon}_{\mathrm{s}}$ en fonction de $\operatorname{Ln} \sigma_{\text {appl }} / G$ permet de déterminer le coefficient de sensibilité de la vitesse de fluage à la contrainte réduite : $n$. On trouve $n$ voisin de 3,5 sur toute la plage de température utilisée (sauf à $293^{\circ} \mathrm{C}$ où l'ordre à courte distance intervient). Ce résultat est en accord avec ceux obtenus sur d'autres phases $\beta$.

Le tracé de $\operatorname{Ln} \stackrel{\circ}{\varepsilon_{\mathrm{s}}}$ à $\sigma_{\text {app } 1} / G$ constante permet de caractériser une valeur constante de $\Delta H$ de l'ordre de $17 \mathrm{kcal} / \mathrm{mole}$. (Excepté pour $\operatorname{Ln} \sigma / G=-7$, ce qui correspond à la valeur forte de $\Delta H_{\text {appl }}$ obtenue à $\sigma_{\text {appl }}=1,8 \times 10 \mathrm{~N} / \mathrm{m}^{2}$ (Fig. 3).)

3. Analyse des résultats. - La valeur de $\Delta H$ obtenue figure 3 peut être comparée à différents modèles en particulier aux deux cas extrêmes envisagés par Brebec et Poirier [7]. Ces auteurs calculent le coefficient de diffusion $\bar{D}$ qui intervient dans le fluage restauration pour une solution solide binaire $\mathrm{AB}$ fortement concentrée. Ils font intervenir les fractions ato-

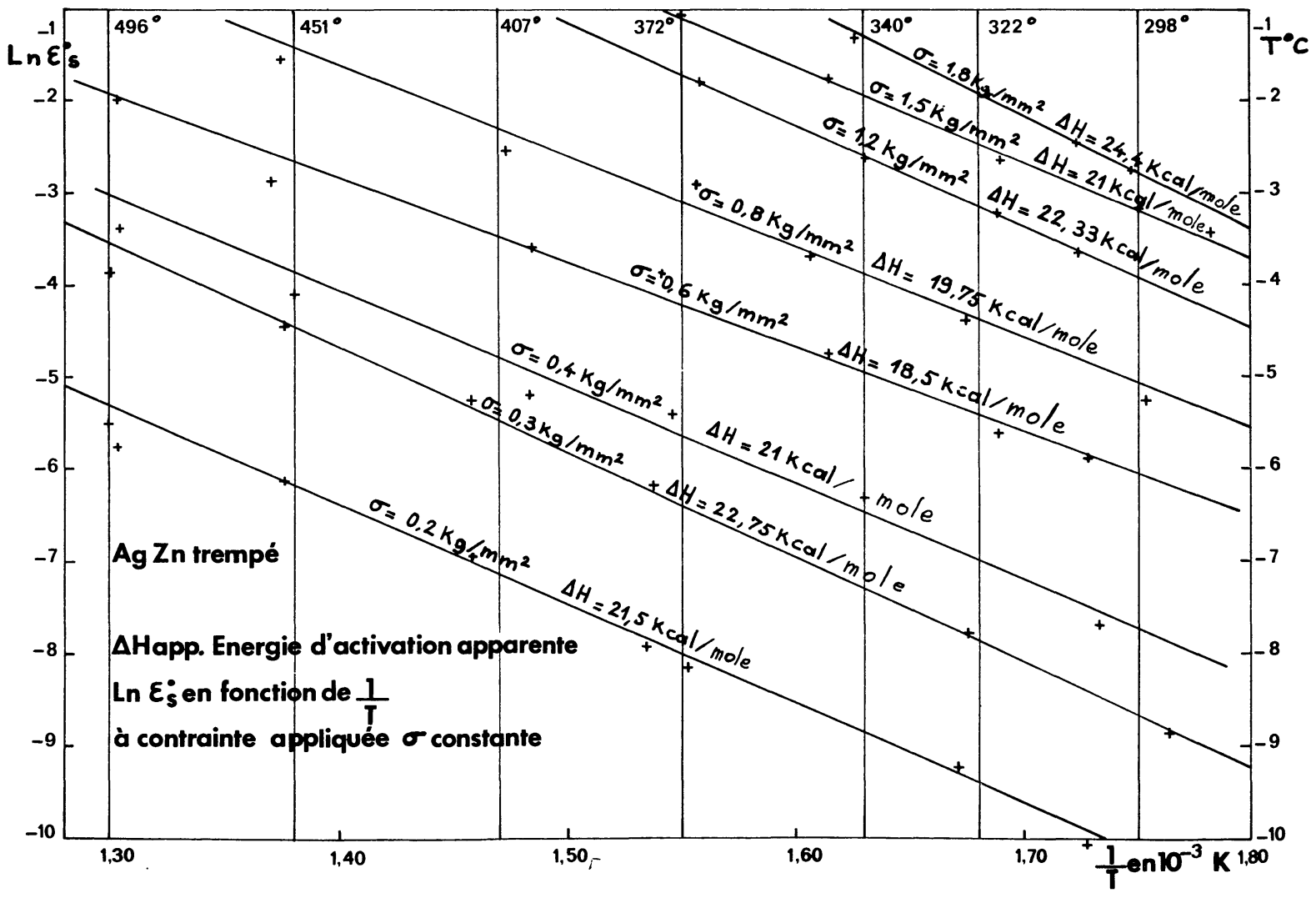

FIG. 1. - Tracé de $\operatorname{Ln} \stackrel{\circ}{\varepsilon_{S}}$ en fonction de $1 / T$ à contrainte appliquée constante. Mesure de l'énergie d'activation apparente.

[Curve of $\operatorname{Ln} \stackrel{\circ}{\varepsilon_{\mathrm{s}}}$ versus $1 / T$ at constant applied stress. Determination of the apparent activation energy.] 


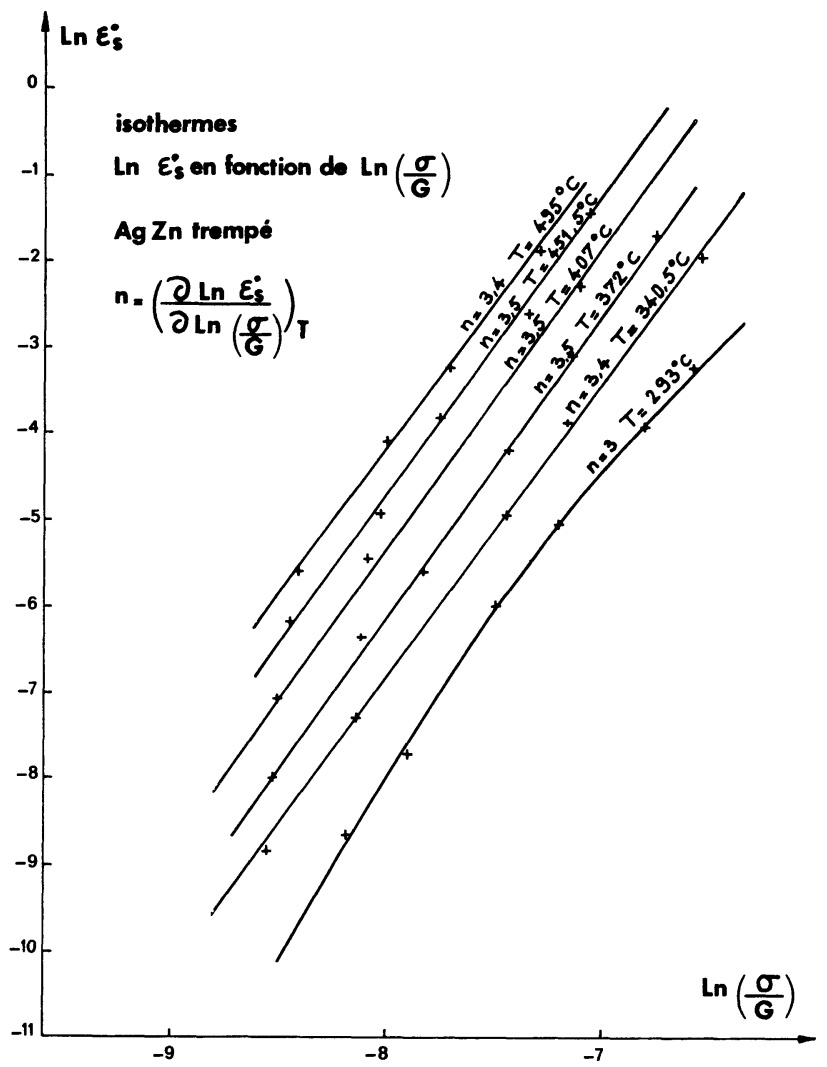

FIG. 2. - Sensibilité de la vitesse de déformation $\stackrel{\triangleright}{\text { s }}_{\text {à }}$ la contrainte réduite $(\sigma / G)$.

[Stress sensitivity of creep rate $\stackrel{\circ}{\varepsilon_{\mathrm{s}}}$ to reduced stress $\sigma / G$.] miques des constituants $n_{\mathrm{A}}$ et $n_{\mathrm{B}}$ et leurs coefficients d'auto-diffusion $D_{\mathrm{A}}$ et $D_{\mathrm{B}}$. Les deux cas envisagés sont :

3.1 Le RÉGime STATIONNAIRE. - Le flux de chacun des constituants est proportionnel à sa fraction atomique, soit avec les notations habituelles

$$
\frac{J_{\mathrm{A}}}{n_{\mathrm{A}}}=\frac{J_{\mathrm{B}}}{n_{\mathrm{B}}}
$$

qui conduit à un coefficient $\bar{D}^{\prime}$ donné par :

$$
\frac{1}{\overline{D^{\prime}}}=\frac{1}{\varphi}\left(\frac{n_{\mathrm{A}}}{D_{\mathrm{A}}}+\frac{n_{\mathrm{B}}}{D_{\mathrm{B}}}\right)
$$

où $\varphi$ est un facteur thermodynamique.

3.2 L'Alliage ReSTe HOMOgÈNe. - On obtient alors un coefficient :

$$
\bar{D}=\varphi\left(n_{\mathrm{A}} D_{\mathrm{A}}+n_{\mathrm{B}} D_{\mathrm{B}}\right)
$$

Dans notre cas $n_{\mathrm{A}}=n_{\mathrm{B}}=\frac{1}{2}$.

Les courbes $\bar{D}$ et $\bar{D}^{\prime}$ ont été tracées avec $\bar{\Phi}=1$. En fait, cela se produit essentiellement si les coefficients d'activité $\gamma_{\mathrm{Ag}}$ et $\gamma_{\mathrm{Zn}}$ sont égaux à 1 . Peu de composés intermétalliques désordonnés présentent ce comportement idéal [9]. Cependant $\bar{\Phi} \neq 1$ se traduit par une simple translation des courbes $\bar{D}$ et $\bar{D}^{\prime}$ si $\varphi$ varie peu (ici on a $\bar{\Phi}$ compris entre 2 et 3 ce qui peut se traduire

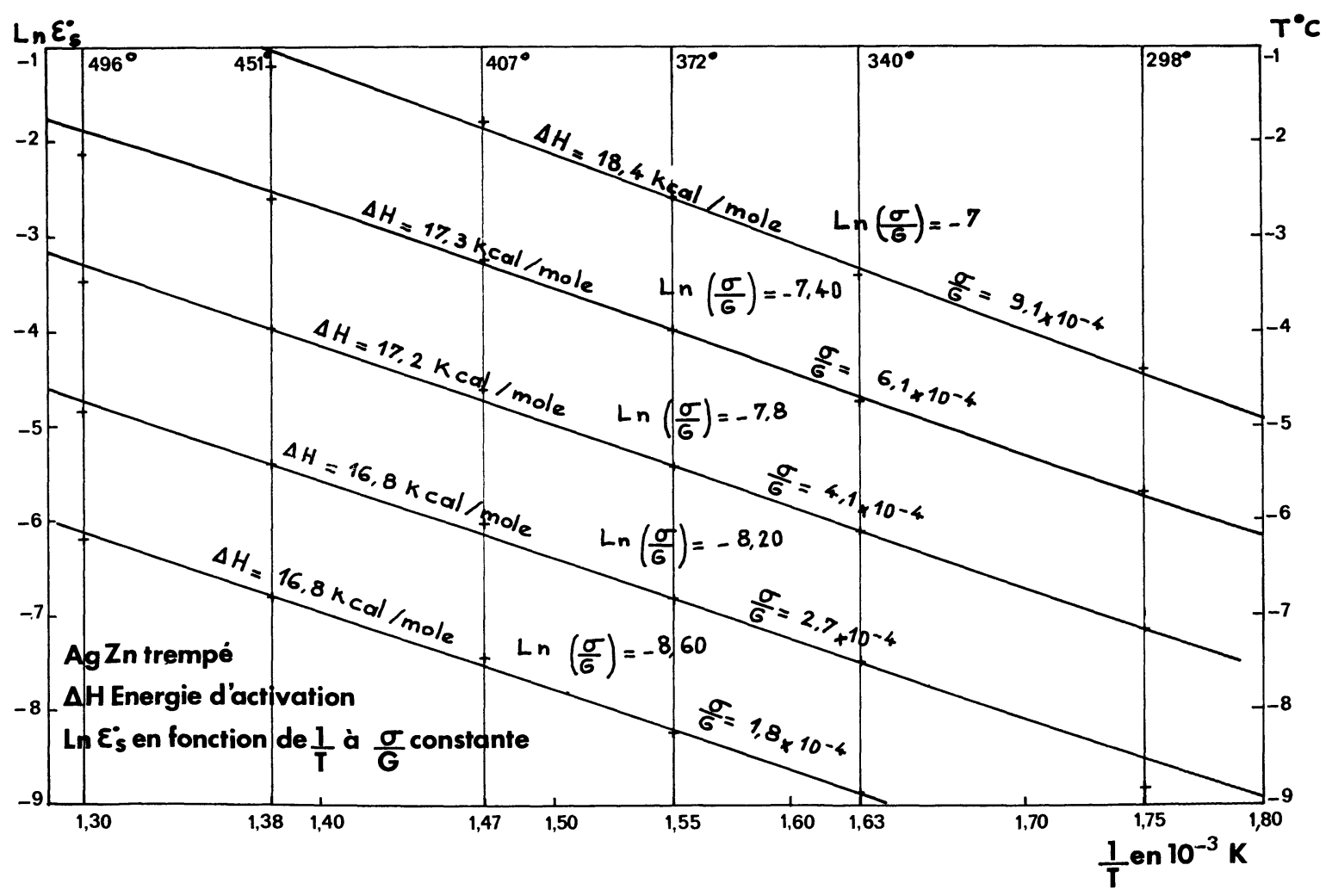

FIG. 3. - Tracé de $\operatorname{Ln} \stackrel{\circ}{\varepsilon_{\mathrm{s}}}$ en fonction de $1 / T$ à contrainte réduite constante. Mesure de l'énergie d'activation.

[Curve of Ln $\stackrel{\circ}{8}_{3}$ versus $1 / T$ at a constant reduced stress. Determination of the activation energy.] 
par une translation d'une unité dans la courbe 4). Poirier et Brebec utilisent le terme

$$
Q c=-R \frac{\partial \operatorname{Ln} \Phi}{\partial \frac{1}{T}} .
$$

On connaît peu de valeurs des potentiels chimiques $\mu_{\mathrm{Zn}}$ et $\mu_{\mathrm{Ag}}$ dans le $\mathrm{Zn}$-Ag équiatomiques donc de $\gamma_{\mathrm{Ag}}$ et $\gamma_{\mathrm{Zn}_{\mathbf{n}}}$. Cependant les valeurs existant pour $\mu_{\mathrm{Zn}}$ [10] permettent de montrer que $Q_{\varphi}$ est de l'ordre de grandeur de l'incertitude expérimentale $\left(Q_{\varphi} \simeq-1 \mathrm{kcal} /\right.$ mole) et peut être négligée.

La comparaison avec l'expérience peut être faite à partir de la figure 4 sur laquelle sont portés les diagrammes d'Arrhénius de $D_{\mathrm{Zn}}$ et $D_{\mathrm{Ag}}$ [8] et de $\bar{D}$ et $\bar{D}^{\prime}$ calculés à l'aide des formules précédentes.

Il est clair que la valeur de $\Delta H$ trouvée étant proche de celle du coefficient le plus petit $D_{\mathrm{Ag}}$, elle doit être comparée à la valeur théorique obtenue en régime stationnaire (Fig. 4). L'élimination du cas homogène est ici rendue possible par l'écart important qui existe entre les énergies d'activation de $D_{\mathrm{Ag}}$ et $D_{\mathrm{Zn}}$. On peut alors constater que $\Delta H$ est très proche de la valeur prévue dans le cas où la vitesse de fluage est contrôlée par la diffusion en volume, le transport de matière par lacune s'effectuant en régime stationnaire.

Le même résultat est obtenu pour le laiton $\beta$ désordonné [3]. Dans ce cas, l'énergie d'activation trouvée en fluage est voisine de celle de la diffusion de $\mathrm{Cu}$ qui a le coefficient le plus petit. Cependant, dans ce dernier cas, on obtient une enthalpie d'activation du fluage plus grande car si $D_{\mathrm{Cu}}<D_{\mathrm{Zn}}$ on a par contre $\Delta H_{\mathrm{Cu}}>\Delta H_{\mathrm{Zn}}$. Cette analyse du fluage repose sur l'idée que la restauration contrôle le fluage. Si l'on utilise l'équation empirique de Dorn :

$$
\frac{\stackrel{\circ}{\varepsilon_{\mathrm{s}}} k T}{D G b}=A_{5}\left(\frac{\sigma}{G}\right)^{n}
$$

en prenant $D=\bar{D}^{\prime}$, les valeurs de $\stackrel{\odot}{\varepsilon}_{\text {s }}$ obtenues pour les différentes températures en contraintes et celles de $G$ mesurées en dynamique, on peut constater que cette équation est bien vérifiée dans toute la plage de nos résultats (Fig. 5).

Les points expérimentaux obtenus par différentes températures et différentes contraintes appliquées, s'alignent bien, la droite moyenne obtenue sur la figure 5 permet de déterminer $n=3,4$ et $\operatorname{Ln} A_{5}=1,18$. Un argument supplémentaire plaide alors en faveur

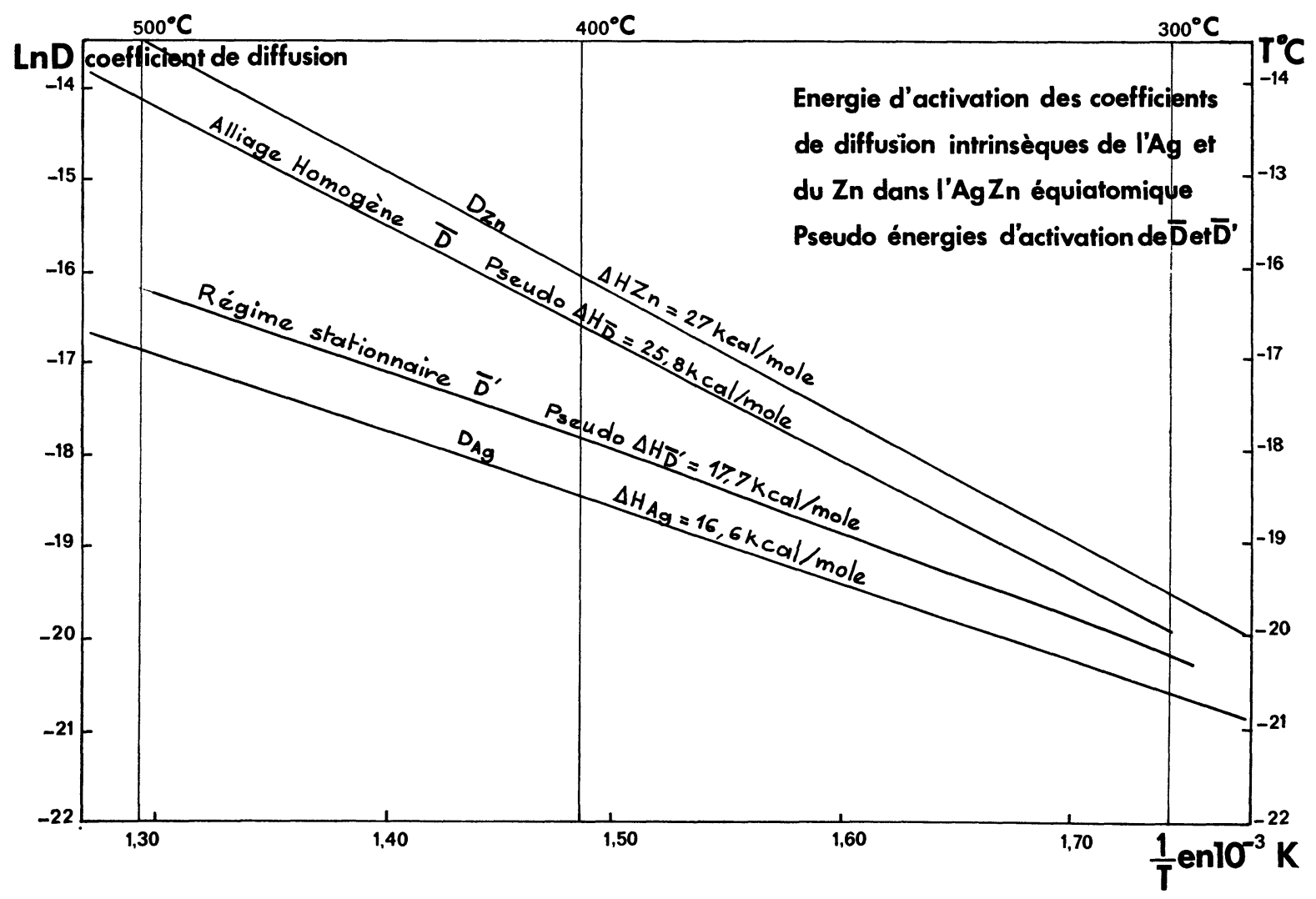

FIG. 4. - Energie d'activation des coefficients de diffusion intrinsèques de l'Ag et du $\mathrm{Zn}$ dans l'Ag-Zn équiatomique. Pseudo-énergies d'activation de $\bar{D}$ et $\bar{D}^{\prime}$.

[Activation energy of the intrinsic diffusion coefficient for $\mathrm{Ag}$ and $\mathrm{Zn}$ in equiatomic $\mathrm{Ag}-\mathrm{Zn}$. Apparent activation energy of $\bar{D}$ and $\bar{D}^{\prime}$.] 


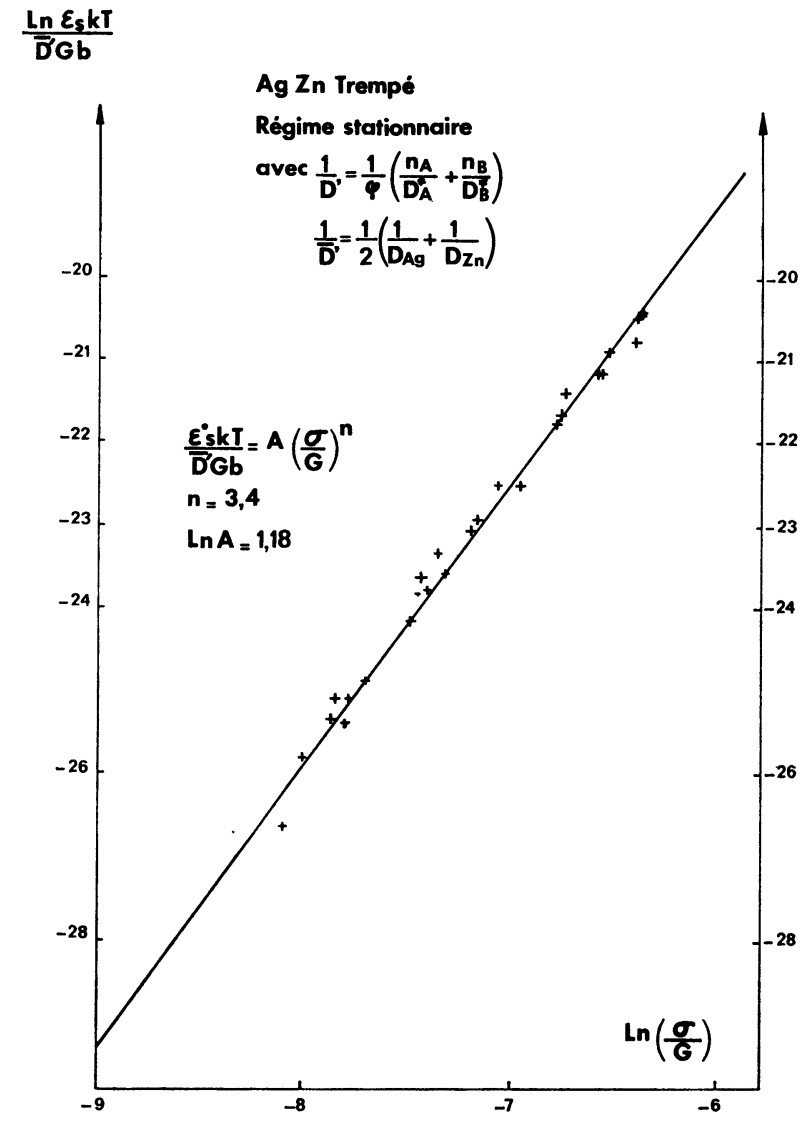

Fig. 5. - Vérification de l'équation généralisée du fluage de Mukherjee, Bird et Dorn.

[Verification of creep generalised equation of Mukherjee, Bird and Dorn.] de l'analyse des coefficients de diffusion proposée plus haut : Stocker et Ashby [9] ont montré qu'il existe une relation empirique $n=3,0+0,3 \log _{10} A_{5}$ entre les coefficients de l'équation de Dorn et ce, par compilation de résultats expérimentaux. Ils ont de plus remarqué que l'équation de Dorn a un sens physique si $A_{5}$ est de l'ordre de 1 . Nous sommes effectivement dans ce cas. La valeur expérimentale de $\operatorname{Ln} A_{5}$ : Ln $A_{5}=1,18$ est en effet extrêmement faible quand on la compare aux valeurs expérimentales de :

$$
\operatorname{Ln} \frac{\stackrel{\circ}{\varepsilon_{\mathrm{s}}} k T}{D G b}
$$

qui sont comprises entre -20 et -27 . Elle reste dans l'intervalle d'incertitude autour de zéro.

4. Conclusion. - L'utilisation d'alliages binaires dont les composants ont des énergies de diffusion suffisamment différentes, peut permettre d'apporter une contribution expérimentale au problème du choix du coefficient de diffusion dans le fluage des alliages à forte concentration. Les résultats expérimentaux montrent, lorsque ce choix doit être fait, que pour les phases $\beta$ il faut utiliser $\bar{D}^{\prime}$. Ce cas correspond à celui où la redistribution des constituants $A$ et $B$ (qui ne s'échangent pas à la même vitesse) est suffisamment rapide pour qu'il s'établisse un gradient de concentration stationnaire en tête des dislocations au cours du fluage.

Ce travail fait partie d'une étude effectuée à l'aide d'une A. T. P. rupture et fluage du C. N. R. S.

\section{Bibliographie}

[1] PoIRIER, J. P., Plasticité à haute température des solides cristallins (Eyrolles edit.) 1976.

[2] SHERBY, O. D., BURKE, P. M., Mechanical behaviour of cristalline solids at elevated temperature, (Perganom Press) 13, no 7, pp. 325-388, 1967.

[3] Delobelle, P., OYtana, C., Mém. Sci. Rev. Métall. 4 (1976) 293.

[4] Saxl, I., Kroupa, F., Phys. Stat. Sol. (a) 11 (1972) 167.

[5] Oytana, C., Delobelle, P., Varchon, D., Revue Phys. Appl. 11 (1975) 159.

[6] MukherJee, A. K., Bird, J. E., DoRn, J. E., Trans. A. S. M. 62 (1969) 155.
[7] Brebec, G., PoIrier, J. P., Acta Met, 23 (1975) 125.

[8] HAGEL, W. C., dans intermetallic coumpounds (J. H. Westbrook edit.) 1967, chap. 20.

[9] Robinson, P. M. et BeVER, M. B., dans intermetallic compounds (J. H. Westbrook edit.) 1967, chap. 3.

[10] KubaschewsKi, O. et Evans, E., La thermochimie en métallurgie (Gauthier-Villars ed.) 1964.

Hultgren, R., Desai, P. D., Hawkins, D. T., GleiSER, M., Selected values of the thermodynamic properties of binary alloys (A. S. M. Edit.) 1973.

[11] Stocker, R. L., Ashby, M. F., Scr. Metall. 7 (1973) 115. 